\title{
Gadolinium Enhanced MR-angiography Results in Patients With Peripheral Arterial Disease: Positive Predictive Value Compared to Surgery
}

\author{
Seyed Rasool Mirsharifi ${ }^{1}$; Morteza Noaparast ${ }^{1, *}$; Mona Khazravi ${ }^{1}$; Hossein Ghanaati ${ }^{2}$; Madjid \\ Shakiba ${ }^{2}$; Amirsina Sharifi ${ }^{2}$ \\ ${ }^{1}$ Department of General Surgery, Imam Khomeini Hospital, Tehran University of Medical Sciences, Tehran, IR Iran \\ 2 Advanced Diagnostic and Interventional Radiology Research Center (ADIR), Tehran University of Medical Sciences, Tehran, IR Iran \\ ${ }^{*}$ Corresponding Author: Morteza Noparast, Department of General Surgery, Imam Khomeini Hospital, Tehran University of Medical Sciences, Tehran, IR Iran. Tel:+98-2166581657, Fax: \\ +98-2161191609, E-mail:dr.m.noparast@gmail.com
}

Received: December 11, 2014; Revised: December 23, 2014; Accepted: December 27, 2014

\begin{abstract}
Background: Peripheral arterial disease(PAD) represents systematic atherosclerosis of great vessels. PAD affects approximately $10-20 \%$ of patients older than 60 years and is associated with high mortality and morbidity rate debilitating individuals' life.

Objectives: To compare the results of Gadolinium enhanced MR-Angiography and surgery in patients suspected to have peripheral arterial disease.

Materials and Methods: In this prospective cohort study, 30 consecutive patients matching the inclusion criteria were enrolled and MRAngiography was performed prior to surgery for each one.

Results: 22 patients were male $(73.3 \%)$ and the mean age was $60.3 \pm 10.6$ years in our study group. The most common artery for cut off and run off was superior femoral artery in both assessments. Proximal section of each artery was the most common anatomical section for cut off and run off. There was a same report of cut off artery by MR-Angiography and surgery (kappa coefficient of agreement was 0.96, Pvalue $<0.001$ ) and positive predictive value was 0.97 (95\% CI: 0.83-0.99).

Conclusions: According to our findings MR-angiography is an appropriate alternative imaging modality for patients suspected to have peripheral arterial disease and it facilitates the early diagnosis proposed by the clinical findings. Also beneficial characteristics of this method such as low exposure to ionizing radiation, repeatability, and low risk of contrast agent-induced nephropathy make it a modality of choice in patients with renal impairment.
\end{abstract}

Keywords:Angiography; Peripheral Vascular Disease; Surgery

\section{Background}

Peripheral arterial disease (PAD) represents systematic atherosclerosis of great vessels (1). PAD affects approximately $10-20 \%$ of patients older than 60 years and is associated with high mortality and morbidity rate debilitating individuals' life. Anatomically, about 30\% of lesions are located in the iliac arteries and $70 \%$ are located in the femoro-popliteal and tibial arteries (2-7). Increased risk of myocardial infarction, stroke and cardiovascular death in these patients, necessitate the appropriate diagnostic and therapeutic strategies. In this regard different imaging modalities have been introduced. Duplex ultrasonography, computed tomographic (CT) angiography, contrast enhanced magnetic resonance (MR) angiography, and digital subtraction angiography (DSA) have been reported to facilitate the exact diagnosis which has been proposed by ankle brachial index measurement and Doppler ultra-sonography (8-14). DSA is currently the modality of choice for evaluating PAD but invasive- ness of procedure, high doses of ionizing radiation exposure and risk of contrast agent-induced nephropathy lead physicians to use MR angiography (8). MR angiography using Gadolinium based contrast provides rapid detailed evaluation of the peripheral arteries. No sedation needed protocols of imaging, lowering ionizing radiation exposure and lowering risk of contrast agentinduced nephropathy made contrast enhanced magnetic resonance (MR) angiography, preferred modality of imaging in many institutes and DSA is reserved for therapeutic rather than diagnostic interventions $(13,15$ 17). However, Gadolinium enhanced MR angiography is not completely safe. Nephrogenic systemic fibrosis (NFS) is known to be complication of Gadolinium based contrast agents in patients with severe renal dysfunction (18-20). Despite the all technological improvements in imaging modalities, definite diagnosis and treatment is still relied on surgical interventions. On the other hand 
surgical intervention is invasive and usually performed in late stages (21-23).

\section{Objectives}

Thus we aimed to compare the accuracy of Gadolinium enhanced MR angiography to surgical intervention in terms of diagnosis of cut-off and run-off of lower limbs arteries in patients with PAD.

\section{Materials and Methods}

This prospective cohort study was held on patients referring to vascular surgery ward of Imam Khomeini hospital complex, Tehran University of Medical Sciences, Tehran, Iran from September 2012 to January 2014. Hospital ethic committee approved our study protocol of human experimentation based on declaration of Helsinki 1975 revision and all patients gave informed consent of participation. We used all data from eligible patients matching inclusion criteria. Patients with following symptoms were consecutively chosen as suspected to have PAD; pain (described as initiation of debilitating feeling in lower extremities preventing to perform daily activity) and limb claudication (inability to walk as daily routine). The first 42 patients referred to our clinic with above symptoms were examined clinically and Ankle-Brachial Index (ABI) was calculated. Weak pulse of lower extremities or coldness and pallor of feet were other confirming symptoms for diagnosis of PAD. Those patients with ABI $<0.9$ were selected to enter study group as if they had not following criteria: having pacemaker or metal parts in their body, severe claustrophobia, and GFR $<30 \mathrm{~mL} / \mathrm{min} / 1.73 \mathrm{~m}^{2}$, which was needed for the application of intravenous Gadolinium based contrast agent. 10 patients were excluded because of high $\mathrm{ABI}$ than preset cut point; one patient was excluded of severe renal impairment and 1 patient for implantation of cardiac pacemaker interfering with MRI function. Thus total number of 30 patients was enrolled to the study.

\subsection{Gadolinium Enhanced MR Angiography}

All imagings were performed in Imam Khomeini medical imaging Center using a clinical 3 Tesla MR system (MAGNETOM Verio, Siemens HealthCare Global, Erlangen, Germany) equipped with high performance gradients strength (40 mT/m @ 200T/m/s). Phased-array coils were used for signal reception. Patients were positioned supine. For gadanilinum enhanced MR-Angiography it took 30-40 minutes and no intravenous sedation was used. A standard three step automated moving-table protocol covering pelvis, thigh, and calf station was used with the following basic parameters: TR/TE, 2.8 $\mathrm{ms} / 1.06 \mathrm{~ms}$; flip angle, $20^{\circ}$; voxel size, $1.7 \times 1.7 \times 3.5 \mathrm{~mm}$. For all examinations, commercially available Gadopentetate Dimeglumine (Magnevist, Schering, Berlin, Ger- many) was used at a concentration of 0.2-0.3 $\mathrm{mmol} / \mathrm{Kg}$. Patients under $75 \mathrm{~kg}$ in body weight received $20 \mathrm{~mL}$ of undiluted Gd, and patients over $75 \mathrm{~kg}$ received $30 \mathrm{~mL}$ Gd. A bolus timing technique was performed using real time MRI of the pelvic station. Upon contrast agent reaching the distal abdominal aorta, manual triggering of the MR angiography acquisition was initiated. The injection protocol was biphasic, in which the first half was administered at a flow rate of $1 \mathrm{~mL} / \mathrm{s}$, and the second half at a flow rate of $0.5 \mathrm{~mL} / \mathrm{s}$, followed by a flush of $30 \mathrm{~mL}$ normal saline solution at a flow rate of $0.5 \mathrm{~mL} / \mathrm{s}$. The raw data was post processed into coronal MIP images and stitched together from each station to form a single image. Two experienced radiologists evaluated the Gadolinium enhanced-MR angiography images. Each radiologist reported separately and was blinded to patient demographics and clinical data, and to each other's results.

\subsection{Surgical Technique}

Revascularization and exploration of lower extremities with PAD was performed intra-operatively following MR-Angiography in order to determine the extent of stenosis. Reversed saphenous vein or synthetic prothesis graft technique was used to construct distal bypass grafting under general anesthesia. Arterial clamping was performed after intravenous injection of unfractionated heparin (5000 IU), and autologous heparinized vein or synthetic prosthesis was transplanted. Surgical findings including cut-off and run-off sites and other relevant pathologies were recorded intra-operatively.

\subsection{Statistical Analysis}

Gadolinium enhanced-MRA reports have been compared to surgical intervention findings (as the goldstandard technique). The rate of agreement between Gadolinium enhanced-MRA and surgery was expressed by calculating the kappa statistic. The kappa statistic is defined as the observed agreement not accounted for by chance. The McNemar test was used to determine if there are differences in report of MRA and surgery about one anatomical section of cut off/run off. All statistical analyses were performed using commercially available software (Statistical Package for Social Sciences, version 16, SPSS Inc. Chicago, Illinois, USA).

\section{Results}

All 30 patients were consecutively selected among patients who met the inclusion criteria. 22 patients (73.3\%) were male and 8 (26.7\%) were female. Mean age was 60.3 \pm 10.6 SD year (ranging from 39 to 77 years). The most common symptom was claudication seen in 20 (66.7\%) patients; other symptoms like ulcer and pain were seen in $7(23.3 \%)$ and $3(10 \%)$ patients, respectively. Accord- 
ing to MRI findings the most common artery for cut off was superior femoral artery (SFA) which accounts for 12 (40\%) patients (Table 1, Figure 1). In our study the most common anatomical section of a single artery for cut off (reported by MRA) was proximal section $(\mathrm{n}=17,56.7 \%)$ (Table 1). Surgical exploration revealed that 11 (36.7\%) patients had cut off in SFA. There was only one disagreement between MRA and surgery findings where MRA reported SFA and surgery reported Common Femoral Artery (CFA) as cut off artery. In all other patients the identical artery was reported and kappa coefficient of agreement between these two assessments was 0.96 ( $P$ value $<0.001)$. Also McNemar test showed there is not any statistically significant difference between MRA and surgery regarding determination of involved artery ( $p$ value $=0.32$ ) (Tables 2 and 3 ). Almost the same anatomical section as MRA report for cut off was seen in each single artery in surgery reports; kappa coefficient of agreement was 0.88 (P value < 0.001) and McNemar test showed not significant differences $(P$ value $=0.99)$. Contrary to this approximately the same results in findings about cut off between two assessments; findings on run off artery and anatomical section were different. MRA reported SFA $(n=11 ; 36.7 \%)$ as the most common artery for run off and proximal section as the most common anatomical section ( $\mathrm{n}=17 ; 56.7 \%)$ (Table 4 ) but surgery was unable to report the run off artery in 2 cases. Although the most frequent artery (SFA, $n=9$ ) and anatomical section (proximal, $\mathrm{n}=16$ ) reported by surgery was the same as MRA (Table 5) (Figures 2 and 3). Positive Predictive Value (PPV) of MRA in determining cut off in involved artery according to above findings is 0.97 (95\% CI: 0.83-0.99).

Table 1. MR-Angiography Findings on Cut Off ${ }^{\mathrm{a}, \mathrm{b}}$

\begin{tabular}{|c|c|}
\hline Artery & Frequency \\
\hline SFA & $12(40)$ \\
\hline Popliteal & $1(3.3)$ \\
\hline Aorta & $6(20)$ \\
\hline CFA & $3(10)$ \\
\hline EIA & $3(10)$ \\
\hline CIA & $5(16.7)$ \\
\hline \multicolumn{2}{|c|}{ Anatomical section } \\
\hline Proximal & $17(56.7)$ \\
\hline Medial & $3(10)$ \\
\hline Distal & $10(33.3)$ \\
\hline
\end{tabular}

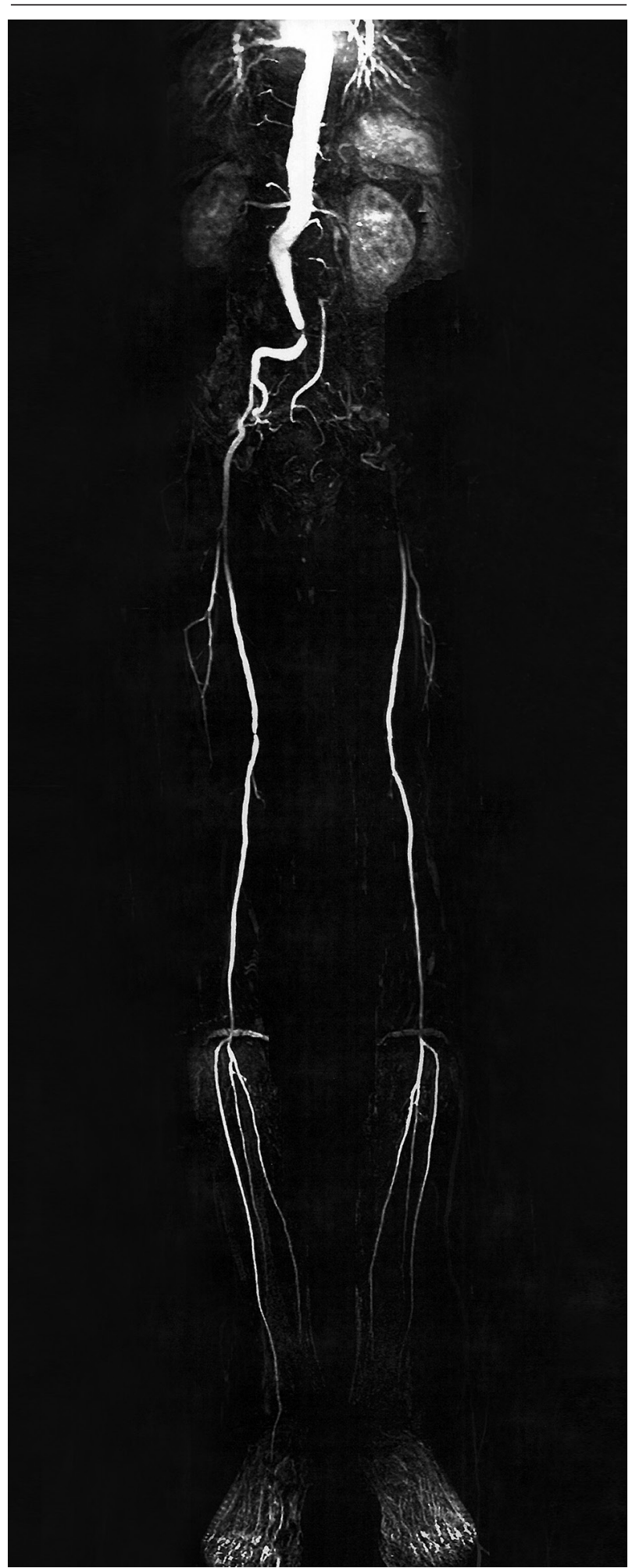

Figure 1. A 71-Year Old Man, MR-Angiography Reported Cut-Off in Bifurcation of Abdominal Aorta and Run-Off in Proximal Common Iliac Artery (CIA) in Right Side and Cut-Off in Bifurcation and Run-Off in Superficial Femoral Artery (SFA) in Left Side, During Surgery in the Right Side, the CutOff Was in Bifurcation and Run-Off in SFA (10 cm After Arterial Origin), and in the Left Side, Cut-Off Was in Bifurcation and Run-Off in SFA. 
Mirsharifi SR et al.

\begin{tabular}{|c|c|}
\hline Artery & Frequency \\
\hline SFA & $11(36.7)$ \\
\hline Popliteal & $1(3.3)$ \\
\hline Aorta & $6(20)$ \\
\hline CFA & $4(13.3)$ \\
\hline EIA & $3(10)$ \\
\hline CIA & $5(16.7)$ \\
\hline \multicolumn{2}{|c|}{ Anatomical section } \\
\hline Proximal & $17(56.7)$ \\
\hline Medial & $3(10)$ \\
\hline Distal & $10(33.3)$ \\
\hline
\end{tabular}

Table 3. Cut Off Artery (MRI) Vs Cut off Artery (Surgery) Cross Tabulation $^{\mathrm{a}}$

\begin{tabular}{lcccc}
\hline Cut Off Artery (MRI) & \multicolumn{4}{c}{ Cut Off Artery (Surgery) } \\
\hline & SFA & Popliteal & Aorta & CFA \\
\hline SFA (n) & 11 & 0 & 0 & 1 \\
\hline Popliteal (n) & 0 & 1 & 0 & 0 \\
Aorta (n) & 0 & 0 & 6 & 0 \\
CFA (n) & 0 & 0 & 0 & 3 \\
EIA (n) & 0 & 0 & 0 & 0 \\
CIA (n) & 0 & 0 & 0 & 0 \\
\hline
\end{tabular}

a Abbreviations: SFA, superior femoral artery; CFA, common femoral artery; EIA, external iliac artery; CIA, common iliac artery.

\begin{tabular}{|c|c|}
\hline Artery & Frequency \\
\hline SFA & $11(36.7)$ \\
\hline ATA & $1(3.3)$ \\
\hline PTA & $4(13.3)$ \\
\hline Popliteal & $5(16.7)$ \\
\hline CFA & $5(16.7)$ \\
\hline Femoral & $3(10)$ \\
\hline CIA & $1(3.3)$ \\
\hline \multicolumn{2}{|c|}{ Anatomical Section } \\
\hline Proximal & $17(56.7)$ \\
\hline Medial & $6(20)$ \\
\hline Distal & $7(23.3)$ \\
\hline \multicolumn{2}{|c|}{ 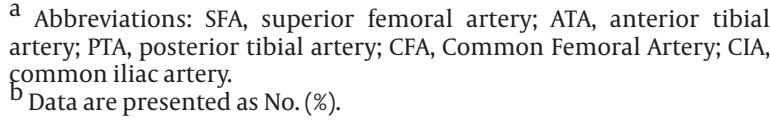 } \\
\hline
\end{tabular}

\begin{tabular}{lc}
\hline \multicolumn{2}{l}{ Table 5. Surgery Findings on Run Off ${ }^{\text {a }}$} \\
\hline Artery & Frequency \\
\hline Negative & $2(6.7)$ \\
SFA & $9(30)$ \\
PTA & $3(10)$ \\
\hline Popliteal & $7(23.3)$ \\
\hline CFA & $6(20)$ \\
Femoral & $3(10)$ \\
\hline Anatomical Section & $2(6.7)$ \\
$\quad$ Negative & $16(53.3)$ \\
$\quad$ Proximal & $5(16.7)$ \\
\hline Medial & $7(23.3)$ \\
\hline Distal & \\
\hline a Abbreviations: SFA, superior femoral artery; PTA, posterior tibial \\
artery; CFA, common femoral artery. b Data are presented as No. (\%).
\end{tabular}

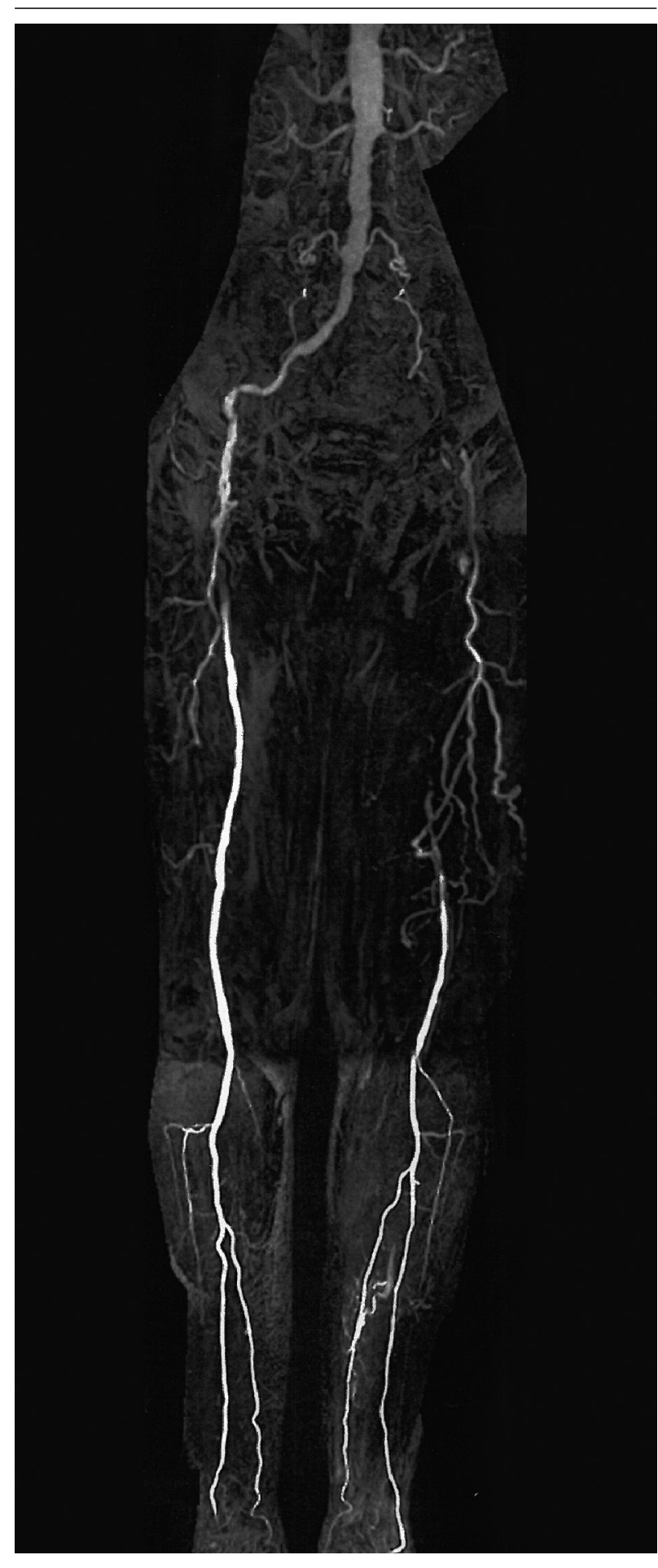

Figure 2. A 72-Year Old Man With Cut-Off in Common Iliac Artery (CIA) Origin and Run-Off in Distal one-Third of Superficial Femoral Artery (SFA) and Deep Femoral Artery (DFA) Located by MR-Angiography, During Surgery the Cut-Off Was in CIA Origin and Run-Off was in Distal of Popliteal Artery

\section{Discussion}

Advanced technological improvements are introduced in field of imaging modalities which changed the old 


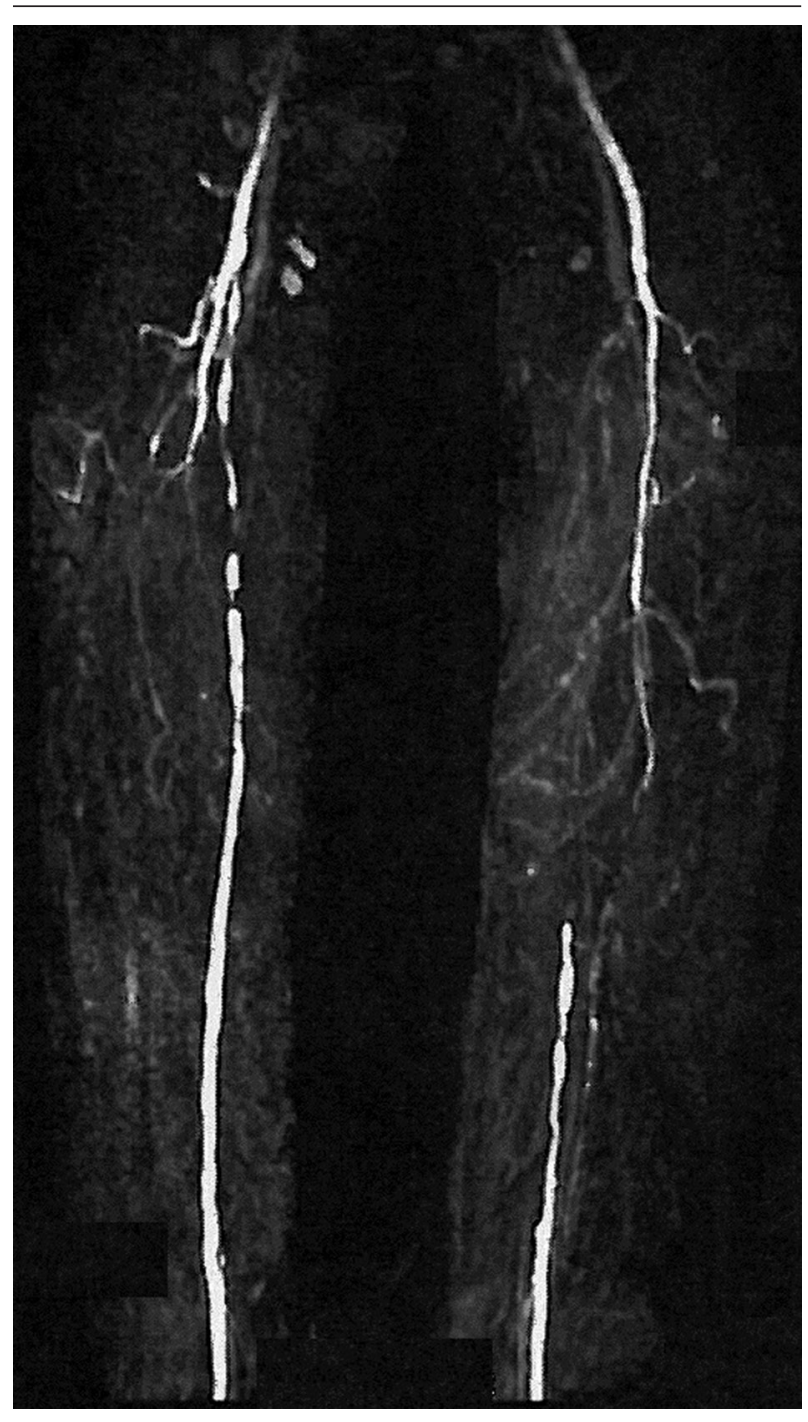

Figure 3. In a 61-Year Old Man, Cut-Off and Run-Off was Located in Superficial Femoral Artery (SFA) and One-Third Distal of Common Femoral Artery (CFA), Respectively by MR-Angiography, During Surgery the Cut-Off and Run Off Was Seen in One-Third Distal of CFA and Proximal of Popliteal Artery, Respectively.

methods of diagnosis (24-26). On the other hand the main question here would be that if these new modalities exactly reflect what happens in the body and how much we can trust on the results provided by these modalities. In this regard we tried to evaluate the predictive value of MRA in means of finding run off and cut off location in patients with PAD and compare what is reported by MRA with what is seen in open surgeries. We found that Gadolinium enhanced MRA is able to report the exact point of cut off and run off in blood flow of lower limb arteries with positive predictive value of 0.97 (95\% CI: 0.83-0.99) regardless of the anatomical position of occlusion. Previous studies emphasized the efficacy of MRA in both contrast agent and conventional methods (19-22, 27); As Binkert et al. found a diagnostic accuracy of
81.9\% in use of calf MRA which significantly is higher than conventional angiography (27). Also Bui and colleagues reported a diagnostic accuracy of 73 to 92 percent for MR-angiography in suspicious cases of PAD (24). Menke et al. performed a review study and reported more than 95 percent of diagnostic accuracy for MR-angiography that is higher than what has been proposed by other investigators (4). Beneficial characteristics of this imaging modality have expanded the application of MRangiography for whole-body imaging in term of exact diagnosis of patients with possible peripheral arterial disease. However some studies have reported more definite results for CT-angiography compared with MRangiography (25-29). Previous study printed by authors of this study (Noaparast et al.) compared 64 multi slice CT angiography and surgery. It has been proposed that CT-angiography (CTA) is capable of reporting anatomical site of occlusion and cut off/run off with accuracy of $89 \%$ and positive predictive value of $96.8 \%$. The same PPV was observed in current study (97\%) by MR-Angiography. It can be concluded that MRA is as definite as CTA in reporting occlusion site and cut off/run off (30). New studies have been dedicated to design new protocols of imaging to maximize the accuracy and lowering the side effects; in this regard Hodnett PA and colleagues reported that quiescent-intervalsingle-shot (QISS) magnetic resonance (MR) angiography as an alternative to imaging modalities for symptomatic PAD in patients whom the administration of iodinated or gadoliniumbased contrast agents is contraindicated (31). In another research Wang J declared that administration of lower dose of contrast agent would not affect the Image quality and diagnostic performance of MRA when $0.1 \mathrm{mmol} / \mathrm{kg}$ gadobenate dimeglumine is used instead of $0.2 \mathrm{mmol} /$ $\mathrm{kg}$ gadopentetate dimeglumine and at least equivalent results are obtained (32). In our study we compared MR-Angiography and surgery in term of finding cut off and run off in lower extremities in patients with PAD and proved the power of MRA in this regard but the fact that our patients were chosen among patients referring to vascular clinic for pain and claudication and the low sample size would be the our limitations. Hence further studies with larger sample size and more powerful statistical analysis among patients referring to general clinics is advised. Nowadays the feasibility and accuracy of MRA in evaluation of lower limbs artery is obvious for every clinicians and advantageous characteristics like low ionizing radiation exposure, the ability to repeat the imaging and low time consuming protocols has convinced the clinicians to applicate this modality instead of DAS.

\section{Acknowledgements}

Authors would like to appreciate the help and support from Imaging Center of Imam Khomeini Hospital technicians. 


\section{Financial Disclosure}

The authors declare that the research was conducted in state of no conflict of Interest and this study was not supported by industrial grants.

\section{Authors' Contributions}

Study concept and design: Noparast, Mirsharifi and Ghanaati Acquisition of data: Khazravi and Shakiba Analysis and interpretation of data: Khazravi, Shakiba and Sharifi Drafting of the manuscript: Sharifi, Shakiba and khazravi Critical revision of the manuscript for important intellectual content: Noparast, Mirsharifi, Ghanaati, Shakiba, Sharifi Statistical analysis: Shakiba and Sharifi Administrative, technical, and material support: Noparast, Ghanaati Study supervision: Noparast, Ghanaati, Mirsharifi. References.

\section{References}

1. Geramizadeh B, Motevalli D, Nikeghbalian S, Malek Hosseini SA. Histopathology of post-transplant liver biopsies, the first report from iran. Hepat Mon. 2013;13(6).

2. Pasternak RC, Criqui MH, Benjamin EJ, Fowkes FG, Isselbacher EM, McCullough PA, et al. Atherosclerotic Vascular Disease Conference: Writing Group I: epidemiology. Circulation. 2004;109(21):2605-12.

3. Hodnett PA, Ward EV, Davarpanah AH, Scanlon TG, Collins JD, Glielmi CB, et al. Peripheral arterial disease in a symptomatic diabetic population: prospective comparison of rapid unenhanced MR angiography (MRA) with contrast-enhanced MRA. AJR Am J Roentgenol. 2011;197(6):1466-73.

4. Menke J, Larsen J. Meta-analysis: Accuracy of contrast-enhanced magnetic resonance angiography for assessing steno-occlusions in peripheral arterial disease. Ann Intern Med. 2010;153(5):325-34.

5. Collins TC, Petersen NJ, Suarez-Almazor M, Ashton CM. The prevalence of peripheral arterial disease in a racially diverse population. Arch Intern Med. 2003;163(12):1469-74.

6. Rowe VL, Lee W, Weaver FA, Etzioni D. Patterns of treatment for peripheral arterial disease in the United States: 1996-2005. J Vasc Surg. 2009;49(4):910-7.

7. Dhangana R, Murphy TP, Coll JR, Ahn SH, Zafar AM, Qadeer FF, et al. Prevalence of abnormal ankle-brachial index among individuals with low or intermediate Framingham Risk Scores. J Vasc Interv Radiol. 2011;22(8):1077-82.

8. Hirsch AT, Haskal ZJ, Hertzer NR, Bakal CW, Creager MA, Halperin JL, et al. ACC/AHA 2005 Practice Guidelines for the management of patients with peripheral arterial disease (lower extremity, renal, mesenteric, and abdominal aortic): a collaborative report from the American Association for Vascular Surgery/Society for Vascular Surgery, Society for Cardiovascular Angiography and Interventions, Society for Vascular Medicine and Biology, Society of Interventional Radiology, and the ACC/AHA Task Force on Practice Guidelines (Writing Committee to Develop Guidelines for the Management of Patients With Peripheral Arterial Disease): endorsed by the American Association of Cardiovascular and Pulmonary Rehabilitation; National Heart, Lung, and Blood Institute; Society for Vascular Nursing; TransAtlantic Inter-Society Consensus; and Vascular Disease Foundation. Circulation. 2006;113(11):e463-654.

9. Lin PH, Bechara C, Kougias P, Huynh TT, LeMaire SA, Coselli JS Assessment of aortic pathology and peripheral arterial disease using multidetector computed tomographic angiography. Vasc Endovascular Surg. 2008;42(6):583-98.

10. Iwai T, Izumi T, Inoue T, Maegawa J, Fuwa N, Mitsudo K, et al. Occipital artery arising from the anterior aspect of the internal carotid artery identified by three-dimensional computed tomography angiography. Iran J Radiol. 2012;9(2):103-5.
11. Shabestari AA, Akhlaghpoor S, Tayebivaljozi R, Fattahi Masrour F. Prevalence of Congenital Coronary Artery Anomalies and Variants in 2697 Consecutive Patients Using 64-Detector Row Coronary CTAngiography. Iran J Radiol. 2012;9(3):111-21.

12. Firouznia K, Ghanaati H, Sharafi A, Abahashemi F, Hashemi $\mathrm{H}$, Jalali AH, et al. Comparing ovarian radiation doses in flat-panel and conventional angiography during uterine artery embolization: a randomized clinical trial. Iran J Radiol. 2013;10(3):111-5.

13. Alswat KA, Sanai FM, Altuwaijri M, Albenmousa A, Almadi M, AlHamoudi WK, et al. Clinical characteristics of patients with hepatocellular carcinoma in a middle eastern population. Hepat Mon. 2013;13(5).

14. Pan FS, Xu M, Wang W, Zhou LY, Xie XY. Infantile hepatic hemangioendothelioma in comparison with hepatoblastoma in children: clinical and ultrasound features. Hepat Mon. 2013;13(8).

15. Lakshminarayan R, Simpson JO, Ettles DF. Magnetic resonance angiography: current status in the planning and follow-up of endovascular treatment in lower-limb arterial disease. Cardiovasc Intervent Radiol. 2009;32(3):397-405.

16. Ersoy H, Rybicki FJ. MR angiography of the lower extremities. AJR Am J Roentgenol. 2008;190(6):1675-84.

17. Nazarpoor M, Poureisa M, Daghighi MH. Comparison of maximum signal intensity of contrast agent on t1-weighted images using spin echo, fast spin echo and inversion recovery sequences. Iran J Radiol. 2012;10(1):27-32.

18. Broome DR, Girguis MS, Baron PW, Cottrell AC, Kjellin I, Kirk GA. Gadodiamide-associated nephrogenic systemic fibrosis: why radiologists should be concerned. AJR Am J Roentgenol. 2007;188(2):586-92.

19. Wertman R, Altun E, Martin DR, Mitchell DG, Leyendecker JR, O'Malley RB, et al. Risk of nephrogenic systemic fibrosis: evaluation of gadolinium chelate contrast agents at four American universities. Radiology. 2008;248(3):799-806.

20. Kuo PH, Kanal E, Abu-Alfa AK, Cowper SE. Gadolinium-based MR contrast agents and nephrogenic systemic fibrosis. Radiology. 2007;242(3):647-9.

21. JL M. Lower extremity arterial disease. In: Cronenwett J, Johnston W editors. Rutherford's Vascular Surgery: Saunders Elsevier; 2010.

22. Creager MA. Peripheral arterial disease. In: Bonow RO, Zipes DP, Libby P editors. Braunwald's Heart Disease: A Textbook of Cardiovascular Medicine.: Saunders; 2011.

23. Ozkan B, Gungor D, Yildirim UM, Harman A, Ozen O, Aytekin C. Endovascular stent placement of juxtaanastomotic stenosis in native arteriovenous fistula after unsuccessful balloon angioplasty. Iran J Radiol. 2013;10(3):133-9.

24. Bui BT, Miller S, Mildenberger P, Sam A, 2nd., Sheng R, Omniscan MRA Investigator Panel. Comparison of contrast-enhanced MR angiography to intraarterial digital subtraction angiography for evaluation of peripheral arterial occlusive disease: results of a phase III multicenter trial. J Magn Reson Imaging. 2010;31(6):1402-10.

25. Diop AD, Braidy C, Habouchi A, Niang K, Gageanu C, Boyer L, et al. Unenhanced 3D turbo spin-echo MR angiography of lower limbs in peripheral arterial disease: a comparative study with gadolinium-enhanced MR angiography. AJR Am J Roentgenol. 2013;200(5):1145-50.

26. Nielsen YJ. Whole-body MR angiography in patients with peripheral arterial disease. Dan Med Bull. 2010;57(12):B4231.

27. Binkert CA, Baker PD, Petersen BD, Szumowski J, Kaufman JA Peripheral vascular disease: blinded study of dedicated calf MR angiography versus standard bolus-chase MR angiography and film hard-copy angiography. Radiology. 2004;232(3):860-6.

28. Ouwendijk R, de Vries M, Pattynama PM, van Sambeek MR, de Haan MW, Stijnen T, et al. Imaging peripheral arterial disease: a randomized controlled trial comparing contrast-enhanced MR angiography and multi-detector row CT angiography. Radiology. 2005;236(3):1094-103.

29. Leiner T, Kessels AG, Nelemans PJ, Vasbinder GB, de Haan MW Kitslaar PE, et al. Peripheral arterial disease: comparison of color duplex US and contrast-enhanced MR angiography for diagnosis. Radiology. 2005;235(2):699-708.

30. Noaparast M, Rabani A, Karimian F, Bodaghabadi M, Aran S, Mir- 


\section{Mirsharifi SR et al.}

sharifi R, et al. Diagnostic Accuracy of Sixty Four Multi-Slice CT Angiography in Assessment of Arterial Cut-Off and Run-Off in Comparison with Surgical Findings. Iran J Radiol. 2011;8(2):89-96.

31. Hodnett PA, Koktzoglou I, Davarpanah AH, Scanlon TG, Collins JD, Sheehan JJ, et al. Evaluation of peripheral arterial disease with nonenhanced quiescent-interval single-shot MR angiography.
Radiology. 2011;260(1):282-93.

32. Wang J, Yan F, Liu J, Lu J, Li D, Luan J, et al. Multicenter, intra-individual comparison of single dose gadobenate dimeglumine and double dose gadopentetate dimeglumine for MR angiography of the peripheral arteries (the Peripheral VALUE Study). J Magn Reson Imaging. 2013;38(4):926-37. 